\title{
Diagnostic reference levels for paediatric computed tomography
}

\begin{abstract}
Authors:
Zakariya Vawda ${ }^{1}$

Richard Pitcher ${ }^{1}$

John Akudugu ${ }^{1}$

Willem Groenewald

Affiliations:

${ }^{1}$ Department of Medical

Imaging and Clinical

Oncology, Stellenbosch

University, South Africa

\section{Correspondence to:}

Zakariya Vawda

Email:

zvawda@vodamail.co.za

Postal address:

PO Box 19139, Tygerberg

7505 , South Africa

Dates:

Received: 25 May 2015

Accepted: 07 Oct. 2015

Published: 30 Nov. 2015

How to cite this article:

Vawda Z, Pitcher R, Akudugu

J, Groenewald W. Diagnostic

reference levels for paediatric

computed tomography. S

Afr J Rad. 2015;19(2); Art.

\#846, 4 pages. http://dx.doi.

org/10.4102/sajr.v19i2.846

\section{Copyright:}

(C) 2015. The Authors.

Licensee: AOSIS

OpenJournals. This work is licensed under the Creative Commons Attribution License.
\end{abstract}

\section{Read online:}

Objectives: To establish local diagnostic reference levels (LDRLs) for emergency paediatric head computed tomography (CT) scans performed at a South African (SA) tertiary-level hospital and to compare these with published data.

Materials and methods: A retrospective analysis was conducted of volume-based CT dose index $\left(\mathrm{CTDI}_{\mathrm{vol}}\right)$ and dose length product (DLP) data from uncontrasted paediatric head CT scans performed in the Trauma and Emergency Unit of a tertiary-level SA hospital from January to June 2013. A random sample of 30 patients in each of 3 age groups $(0-2,>2-5$ and $>5-10$ years) was used. LDRL values were compared with several national DRLs from Europe and Australia.

Results: Mean CTDI ${ }_{\mathrm{vol}}$ and DLP values were: $30 \mathrm{mGy}$ and $488 \mathrm{mGy} . \mathrm{cm}$ for the $0-2$ years age group; $31 \mathrm{mGy}$ and $508 \mathrm{mGy} . \mathrm{cm}$ for the $>2-5$ years group, and $32 \mathrm{mGy}$ and $563 \mathrm{mGy} . \mathrm{cm}$ for the $>5-10$ years group, respectively. The mean DLP for $0-2$ year-olds was the only parameter outside the range of corresponding published reference data. Stratification into narrower age groupings showed an increase in DLP values with age.

Conclusion: An institutional review of the head CT scanning technique for emergency studies performed on children less than 2 years of age is recommended. The current study highlights the role of LDRLs in establishing institutional dosimetry baselines, in refining local imaging practice, and in enhancing patient safety. Standard age stratification for DRL and LDRL reporting is recommended.

\section{Introduction}

There is a burgeoning global demand for computed tomography (CT). Compared with plainfilm radiography, $\mathrm{CT}$ accounts for relatively large doses of ionising radiation, with $\mathrm{CT}$ exposure currently representing the largest manmade contribution of absorbed dose to the general population. Monitoring of CT radiation dosage is therefore of increasing importance, especially in paediatric imaging, as children are more vulnerable to the harmful effects of ionising radiation; this is particularly true in low- and middle-income countries where there may be constraints on equipment upgrades and maintenance. .,2,3,4,5,6 $^{-1}$

As with all diagnostic studies, CT scans should be clinically justified, provide potential patient benefit and utilise appropriate imaging protocols, to keep radiation doses as low as reasonably achievable (ALARA). ${ }^{3}$ With the latter in mind, the International Commission on Radiological Protection (ICRP) introduced the concept of diagnostic reference levels (DRLs) in 1996.,7 DRLs are accepted as the standard tool to enable optimisation of absorbed dose delivered to the patient undergoing X-ray imaging. These are intended to monitor radiation dose for specific procedures, set the bar for good clinical practice, identify 'outliers' with unacceptably high radiation doses, allow comparison of equipment and protocols, and provide a mechanism for fine-tuning absorbed doses. ${ }^{8}$

National DRLs for specific examinations and patient groups are based on dose distributions observed in national surveys, with the third quartile (the level below which $75 \%$ of all dose data falls) most commonly adopted. Conversely, by definition, $25 \%$ of sampled dose data will be above the DRL, thereby identifying relatively high dosage. ${ }^{8}$ DRLs may also be established at a local or practice level. Such local DRLs (LDRLs) represent typical dosage for a specific examination at a single institution and usually represent the mean of the local distribution, rather than the third quartile. ${ }^{9}$ Ideally, LDRLs should be reviewed frequently, to allow refinement of examination techniques and ongoing radiation dose reduction, whilst maintaining satisfactory image quality. ${ }^{10}$ 
For CT examinations, DRLs are defined in terms of two established dose indicators, namely, volume-based computed tomography dose index $\left(\mathrm{CTDI}_{\mathrm{vol}}\right)$ and dose length product (DLP). The CTDI ${ }_{\mathrm{vol}}$ represents the average dose per slice, whilst the DLP reflects the total energy absorbed along the scan length, and is the product of the CTDI ${ }_{\mathrm{vol}}$ and scan length. ${ }^{1}$ At the conclusion of each study, modern CT scanners

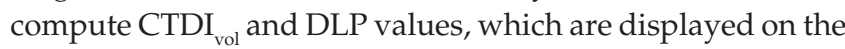
CT workstation and stored with the study images in DICOM format on a picture archiving and communication system (PACS). ${ }^{2}$

In high-income countries such as those in Europe, North America and Australasia, governments have introduced regulations requiring the establishment and maintenance of DRLs in all radiological clinics and practices. However, in low- and middle-income countries, the establishment of DRLs for X-ray imaging practices has not been widely implemented, mainly owing to the constraints under which radiological professionals operate. ${ }^{11}$

Although many countries and institutions are implementing quality assurance measures that include establishment of DRLs for common imaging examinations, DRLs have not played a meaningful role in evaluating the quality and safety of South African (SA) radiological services. There is currently no published national DRL data for South Africa.

The aim of the present study was to establish and describe LDRLs for emergency paediatric head CT scans at a tertiarylevel South African hospital, and to compare these with DRL data published in Europe and Australia. ${ }^{12,13,14,15}$

\section{Methodology}

\section{Setting}

The study was conducted at a 1386-bed tertiary-level SA teaching hospital, which performs approximately 1130 paediatric head CT scans annually. All paediatric emergency scans are performed on the hospital's Trauma and Emergency Unit's scanner, a Somatom Emotion ${ }^{\mathrm{TM}} 6$ multidetector CT scanner (MDCT) (Siemens, Erlangen, Germany), which utilises automatic tube current modulation (CARE Dose ${ }^{\mathrm{TM}} 4 \mathrm{D}$, Siemens, Erlangen Germany). The primary objective of this modulation is to maintain consistent image quality by compensating for patient size and differential attenuation within the scanned body part. ${ }^{9}$ The study was approved by the institutional Health Research and Ethics Committee.

\section{Patient population}

A retrospective audit was undertaken of all uncontrasted emergency paediatric head CT scans performed from January to June 2013. Patients up to 10 years of age were included and stratified by age into groups of younger than 2 years; $>2-5$ years; and $>5-10$ years. In the hospital where the study was conducted, the scan protocol for children older than 10 years was determined by patient size. Some children older than 10 were therefore scanned on an adult protocol. To maintain uniformity, this latter age group was therefore excluded. Random samples of 30 patients were evaluated in each age group..$^{9,10}$

\section{CT protocol and audit}

All scans were performed according to the standard institutional Trauma and Emergency Unit paediatric head CT protocol, which images the brain and upper cervical spine, up to and including the level of C2. Scan parameters were based on a scanner-specific $16-\mathrm{cm}$ diameter plastic phantom and are listed in Table 1. The CTDI vol $_{\text {and DLP values for }}$ each study were recorded on a customised spreadsheet. Scan length was derived from the quotient of the DLP and $\mathrm{CTDI}_{\mathrm{vol}^{*}}{ }^{2}$

Parameters such as tube potential, beam collimation and pitch are specific to the scanning protocol and are outlined in Table 1 . In the present study, the scan reference was set at 230 $\mathrm{mAs}$ for the paediatric protocol (based on a 16-cm diameter phantom). The reference $\mathrm{mAs}$ is a user-specified parameter that drives the automatic adjustment of the tube current, and is usually preset by the vendor application specialist.

For interdisciplinary quality audit purposes, local DRLs were established by calculating mean CTDI $_{\text {vol }}$ and DLP values for each age group. Comparisons with international values were performed using published DRL data from Australia, Switzerland, Germany and the United Kingdom (UK). ${ }^{12,13,14,15}$

\section{Results}

Results obtained in the study are summarised in Table 2. Mean CTDI $_{\text {vol }}$ values were relatively constant across the age groups, ranging from 30 to $32 \mathrm{mGy}$. Mean DLP values increased with patient age from 488 to $563 \mathrm{mGy} . \mathrm{cm}$.

Comparison with published DRLs showed that mean CTDI ${ }_{\mathrm{vol}}$ values were either lower than, or within the range of, other published third quartile national DRLs. ${ }^{12,13,14,15}$

For patients under 2 years of age, the mean DLP value (488 mGy.cm) exceeded the third quartile DLP values

TABLE 1: Summary of scan parameters of the Somatom Emotion ${ }^{\mathrm{TM}} 6$ multi-detector CT scanner used in the present study.

\begin{tabular}{lc}
\hline Parameter & Value \\
\hline Voltage $(\mathrm{kVp})$ & 110 \\
$\mathrm{Q}_{\text {ref }}(\mathrm{mAs})^{\dagger}$ & 230 \\
Rotation time (s) & 1.5 \\
Acquisition (mm) & $6 \times 1.0$ \\
Slice collimation (mm) & 1.0 \\
Slice width (mm) & 5.0 \\
Feed/rotation (mm) & 2.4 \\
Pitch factor & 0.85 \\
Increment & 5.0 \\
\hline ', Qref is the imaging quality reference mAs, which is specific to Siemens (Erlangen, Germany) \\
that is used for automatic tube current modulation (CARE Dose"'4D, Siemens). The value can \\
be adjusted based on image quality requirements and the amount of noise acceptable in the \\
image. It is defined in terms of the effective mAs (actual mAs divided by pitch).
\end{tabular}


TABLE 2: Comparison of mean CTDIvol (mGy) $)^{\dagger}$ and DLP (mGy.cm) ${ }^{\ddagger}$ in the present study (2013), with international diagnostic reference levels (Australia, 2012 $2^{12}$; Switzerland, 2005'3; Germany, 2006 ${ }^{14}$; and the UK, 2003 ${ }^{15}$ ). The year indicates the last year of data collection in each of the studies. All values are relative to the mean values from the present study. ${ }^{\$ 12,13,14,15}$ Ranges of corresponding values are in parentheses.

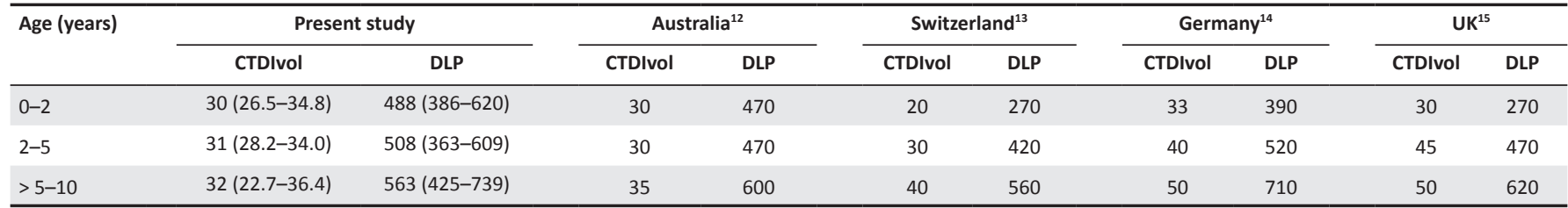

$\dagger$, Volume-based computed tomography dose index.

$\$$, Dose-length product.

$\S$. Age ranges were averaged and approximated to the current study, owing to international variation.

(270 mGy.cm - 390 mGy.cm) of studies from Australia, Switzerland, Germany and the UK, whilst DLP values for older children fell within the corresponding range. ${ }^{12,13,14,15}$ The average scan lengths were $16.5 \mathrm{~cm}$ for the $0-2$ years age group, $16.6 \mathrm{~cm}$ for the $>2-5$ years group, and $17.8 \mathrm{~cm}$ for the $>5-10$ years group, and showed an expected increase with age of patients.

\section{Discussion}

The finding that the mean institutional CTDI $_{\text {vol }}$ values, as listed in Table 2, compare favourably with those published for Australia, Switzerland, Germany and the UK ${ }^{12,13,14,15}$ provides reassurance that the technical parameters of the scans and clinical protocols conform to international standards. This serves as reassurance that effective institutional radiation optimisation practises were applied.

In the newborn to 2-years-old age category, the mean DLP was higher than the 75th percentile for the DRLs reported elsewhere. ${ }^{12,13,14,15}$ This may be a reflection of a suboptimal radiographic technique, because DLP is proportional to scan length. For instance, the local mean scan length in the $0-2$ years age group was found to exceed the range of scan lengths of $9.0-13.5 \mathrm{~cm}$ noted in the European and Australian studies. ${ }^{12,13,14,15}$ It is certainly difficult to discern fine anatomical detail of the cranio-cervical junction and upper cervical spine on the lateral scout projections for CT head scans in young children. However, in the future, more careful attention will be paid when planning CT head scans in young children at our institution, to ensure that only the upper two cervical segments are included. Furthermore, the justification for inclusion of the upper cervical segments in emergency CT head scans in very young children could be reviewed at the institutional level, to assess the positive yield of cervical injury. ${ }^{16}$ The observation that DLP values for the $>2-5$ and $>5-10$ years groups were comparable to those reported in the Australian, Swiss, German and UK studies may be attributed to similarity in scan lengths. ${ }^{12,13,14,15}$

The comparative component of the present study was limited by lack of international uniformity in age stratification for DRL data. We advocate standardised age stratification to facilitate interpretation and comparison of data. With this in mind, CTDI $_{\text {vol }}$ and DLP values from our study were plotted against age in 1-year age increments (Figure 1). CTDI values showed small increases to age 4 years, and then

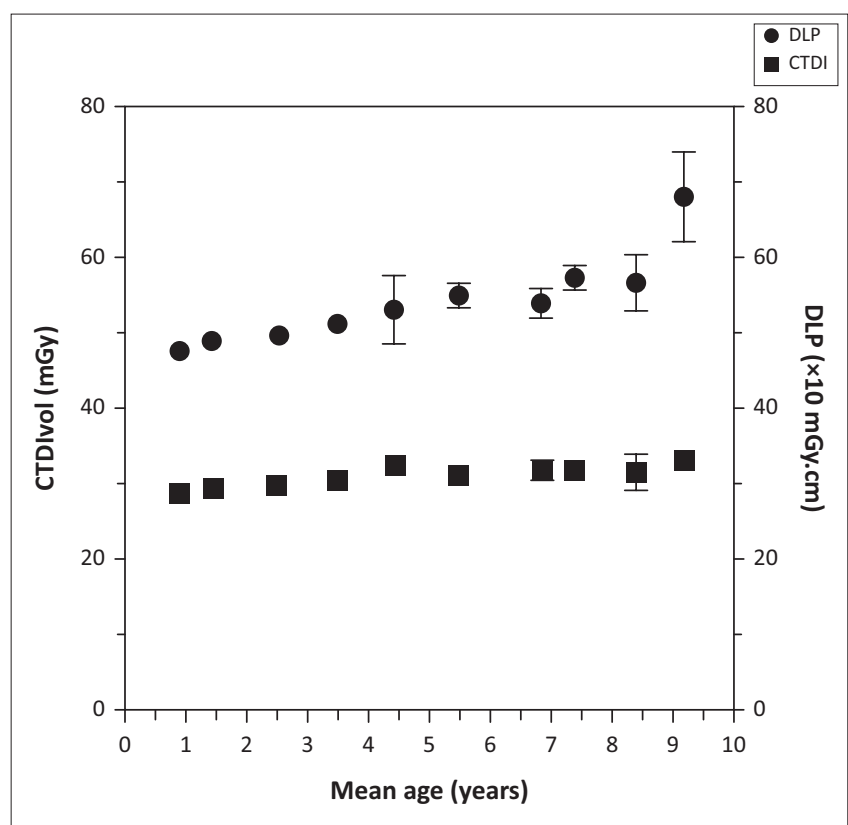

$\mathrm{CTDI}_{\text {vol }}$ (mGy), Volume-based computed tomography dose index; DLP, Dose-length product. FIGURE 1: Mean values of CTDIvol and DLP of paediatric head CT patients in the present study, against mean age for the age groups $<1,1-<2,2-<3,9-10$ years, using a Somatom Emotion ${ }^{\text {TM } 6}$ multi-detector CT scanner. Error bars represent the standard error of the mean.

stabilised. For CTDI ${ }_{\text {vol' }}$ stratification beyond 4 years of age is apparently not required, whilst narrow groupings may be appropriate below 4 years of age. DLP values increase with age for the entire study group (Figure 1). It thus appears that the broad age groupings currently reflecting DLP data in the international literature merit review.

As CTDI ${ }_{\text {vol }}$ and DLP are standard parameters computed for each examination on all modern scanners, they represent a readily available resource for ongoing comparative quality assurance at local, regional, national and international levels. ${ }^{9,10}$ A review of the literature showed that this is the first dedicated local CT DRL survey in Southern Africa. It is hoped that this will be the first of many such audits, and will encourage other radiologists, medical physicists and radiobiologists on the continent, and in resource-limited healthcare settings globally, to initiate such interdisciplinary quality assurance audits. Furthermore, it is envisaged that the data reported here will form the basis of further collaborative work in South Africa with a view to establishing examinationspecific national DRLs for both children and adults. 


\section{Conclusion}

The present study successfully established local DRLs for emergency paediatric CT scans in a hospital operating under resource-constrained conditions. Excellent agreement of these baseline DRLs with international values attested to the safety and efficiency of institutional practice. Furthermore, the role of LDRLs in establishing institutional dosimetry baselines that would optimise local imaging practice, and enhance patient safety, is also highlighted. If a multinational survey of several South African hospitals were to follow the present article, with inclusion of teenagers and adults, it may provide sufficient appropriate data to establish national DRLs. Standard international age stratification for paediatric DRLs are recommended.

\section{Acknowledgements}

The authors give special thanks to IT specialist, Deborah Purdy, for assistance in data mining from the Tygerberg Hospital PACS, and Anne-Marie du Plessis for valuable input regarding clinical protocols for paediatric CT at Tygerberg Hospital.

\section{Competing interests}

The authors declare that they have no financial or personal relationships which may have inappropriately influenced them in writing this article.

\section{Authors' contributions}

Z.V. (Stellenbosch University) was project leader, wrote the study protocol, obtained ethical approval, collected data, analysed the data and wrote the article. R.D.P. and J.M.A. (Stellenbosch University) contributed to design, reviewed and gave input on the final article. W.G. (Stellenbosch University) was the supervisor, reviewed and gave input on the final article.

\section{References}

1. American Association of Physicists in Medicine. Report of AAPM task group 23. The measurement, reporting, and management of radiation dose in CT. College Park, MD: American Association of Physicists in Medicine; 2008.

2. Coursey CA, Frush DP. CT and radiation: What radiologists should know? Appl Radiol. 2008;37:22-29.

3. Sadetzki S, Mandelzweig L. Childhood exposure to external ionising radiation and solid cancer risk. Br J Cancer. 2009;100:1021-1025. PMID: 19337255, http://dx.doi. org/10.1038/sj.bjc.6604994

4. European Commission. Guidance on diagnostic reference levels (DRLs) for medical exposures. c1999 [cited 2014 Apr 25]. Available from: http://ec.europa.eu/energy/ nuclear/radiation_protection/doc/publication/109_en.pdf

5. International Commission on Radiological Protection (IRCP). Radiological protection in paediatric diagnostic and interventional radiology. IRCP publication 121. c2013 [cited 2014 Apr 25]. Available from: http://www.icrp.org/publication. asp?id=ICRP\%20Publication \%20121

6. Brenner DJ, Hall EJ. Computed tomography - An increasing source of radiation exposure. N Engl J Med. 2007;357:2277-2284. PMID: 18046031, http://dx.doi. org/10.1056/NEJMra072149

7. International Commission on Radiological Protection (IRCP). Avoidance of radiation injuries from medical interventional procedures. IRCP publication 85. c2001 [cited 2013 Nov 30]. Available from: http://www.icrp.org/docs/2001_ann_rep.pdf

8. Brady Z, Ramanauskas F, Cain TM, Johnston PN. Assessment of paediatric CT dose indicators for the purpose of optimisation. Br J Radiol. 2012;85:1488-1498. PMID: 22844033, http://dx.doi.org/10.1259/bjr/28015185

9. Institute of Physics and Engineering in Medicine (IPEM). Guidance on the establishment and use of diagnostic reference levels for medical X-ray examinations. IPEM report 88. c2004 [cited 2013 Nov 30]. Available from: http://www.ipem.ac.uk/ Publications/IPEMReportSeries.aspx

10. Al Suwaidi JS, Al Balooshi LG, Al Awadhi HM, et al. Continuous monitoring of CT dose indexes at Dubai Hospital. Am J Roentgenol. 2013:201:858-864. PMID: 24059376, http://dx.doi.org/10.2214/AJR.12.10233

11. World Bank Countries and Economies. c2014 [cited 2014 Nov 31]. Available from: http://data.worldbank.org/country

12. Australian Radiation Protection and Nuclear Safety Agency (ARPANSA). Australian paediatric diagnostic reference levels for MDCT. c2013 [cited 2014 Apr 30]. Available from: http://www.arpansa.gov.au/services/ndrl/paediatric.cfm

13. Verdun FR, Gutierrez D, Vader JP, et al. CT radiation dose in children: A survey to establish age-based diagnostic reference levels in Switzerland. Eur Radiol. 2008;18:1980-1986. PMID: 18389242, http://dx.doi.org/10.1007/s00330-0080963-4

14. Galanski M, Nagel HD, Stamm G, Paediatric CT exposure practice in the Federal Republic of Germany: Results of a nationwide survey in 2005-2006. Hannover: Medizinische Hochschule. c2007 [cited 2014 Apr 30]. Available from: https://www. mhhannover.de/fileadmin/kliniken/diagnostische radiologie/download/Report German_Paed-CT-Survey_2005_06.pdf

15. Shrimpton PC, Hillier MC, Lewis MA, Dunn M. National survey of doses from CT in the UK: 2003. Br J Radiol. 2006;79:968-980. PMID: 17213302, http://dx.doi. org/10.1259/bjr/93277434

16. Strauss KJ, Goske MJ, Kaste SC, et al. Image gently: Ten steps you can take to optimize image quality and lower CT dose for paediatric patients. Am J Roentgenol. 2010:194:868-873. PMID: 20308484, http://dx.doi.org/10.2214/AJR.09.4091 